Editor's Note: These short, critical reviews of recent papers in the Journal, written exclusively by graduate students or postdoctoral fellows, are intended to summarize the important findings of the paper and provide additional insight and commentary. For more information on the format and purpose of the Journal Club, please see http://www.jneurosci.org/misc/ifa_features.shtml.

\title{
Anxious to Drink: Gabapentin Normalizes GABAergic Transmission in the Central Amygdala and Reduces Symptoms of Ethanol Dependence
}

\author{
Kelly J. Clemens* and Leandro F. Vendruscolo* \\ Centre National de la Recherche Scientifique-Unité Mixte de Recherche 5227, Equipe Neuropsychopharmacologie de l'addiction, Université Victor Segalen \\ Bordeaux 1 et 2, 33076 Bordeaux, France \\ Review of Roberto et al. (http://www.jneurosci.org/cgi/content/full/28/22/5762)
}

\begin{abstract}
Alcoholism is a chronic relapsing disorder with up to $80 \%$ of alcohol-dependent individuals who do not receive any form of treatment returning to excessive alcohol consumption. During early abstinence, alcoholics experience psychological and physical symptoms of withdrawal such as anxiety, tremors, and convulsion. Currently these symptoms are controlled with benzodiazepines; however, these drugs are less suitable for long-term use because of a high propensity for abuse, direct interactions with alcohol, the possibility of sensitization to subsequent episodes of withdrawal, and an increased risk of later relapse.
\end{abstract}

Negative affective symptoms, such as anxiety and depression, can persist for extensive periods after acute alcohol withdrawal, and may be integral to the relapse of alcohol drinking as alcoholics attempt to suppress feelings of anxiety through the use of alcohol (Koob, 2003). Currently

Received June 25, 2008; revised July 21, 2008; accepted July 21, 2008

K.J.C. is supported by a postdoctoral fellowship from Association pour la Recherche sur le Nicotianées, France. L.F.V has a postdoctoral fellowship from Fondation Fyssen, France, and received financial support from Institut de Recherches Scientifiques sur les Boissons, France. We thank Drs. M. Cador, S. Ahmed, and M. Darnaudéry for comments on this review.

*K.J.C. and L.F.V. contributed equally to this work.

Correspondence should be addressed to Dr. Leandro F. Vendruscolo, Centre National de la Recherche Scientifique-Unité Mixte de Recherche 5227, Université Victor Segalen Bordeaux 1 et 2, 146 rue Léo Saignat, 33076 Bordeaux cedex, France. E-mail: vendruscolo@hotmail.com.

DOI:10.1523/JNEUROSCI.2928-08.2008

Copyright $\odot$ 2008 Society for Neuroscience $\quad$ 0270-6474/08/289087-03\$15.00/0 there are few successful treatments available for alcohol-dependent individuals after a period of abstinence. Because the majority of alcoholics relapse weeks to months after initial drinking cessation, there is a clear need for the development of safe and efficacious medications for both acute detoxification and protracted abstinence.

Gabapentin, a nonbenzodiazepine anticonvulsant GABA analog, has shown some recent promise in the treatment of alcoholism. Preliminary clinical data suggest that gabapentin may be effective in reducing symptoms of acute alcohol withdrawal and some symptoms of protracted abstinence (Furieri and NakamuraPalacios, 2007). The precise mechanism of action of gabapentin in the alcoholic brain is still unclear. In a recent study published in The Journal of Neuroscience, Roberto et al. (2008) investigated the ability of gabapentin to modulate GABA transmission in the central amygdala (CeA), a brain region known to be implicated in the modulation of emotionality and drug intake (Koob, 2003). In addition, Roberto et al. (2008) explored the effects of gabapentin on altering ethanolrelated behaviors through using animal models of ethanol dependence.

Roberto et al. (2003) have previously reported that acute ethanol application to rat brain slices increases GABAergic transmission in the CeA, at both presynaptic and postsynaptic sites. An ethanol-induced increase in amplitude of GABAergic IPSCs was found to be dependent on postsynaptic $\mathrm{GABA}_{\mathrm{A}}$, but not $\mathrm{GABA}_{\mathrm{B}}$, receptors. This enhanced activation was accompanied by an increase in spontaneous mini-IPSCs and a decrease in paired-pulse facilitation, both indicators of greater presynaptic GABA release. Overall, ethanol appears to increase the responsiveness of receptors to exogenous GABA and reduce the total activity output of the CeA. The precise mechanisms underlying the interaction between ethanol and GABA receptors are not clear.

In the current study (Roberto et al., 2008), superfusion of brain slices with gabapentin produced an electrophysiological response in CeA GABAergic neurons that was similar in direction and amplitude to that detected after application of ethanol [Roberto et al. (2008), their Fig. $2 B$ (http://www.jneurosci.org/cgi/ content/full/28/22/5762/F2)]. However, ethanol and gabapentin appeared to exert their effects via separate mechanisms: the increased GABA transmission evoked by gabapentin was blocked by application of a $\mathrm{GABA}_{\mathrm{B}}$ antagonist, and coapplication of gabapentin and ethanol led to an additive (rather than competitive) effect on GABA transmission [Roberto et al. (2008), their Fig. 2 (http://www.jneurosci.org/cgi/ content/full/28/22/5762/F2)]. These results suggest that in the naive rat, gabapentin and ethanol produce similar effects 
of increasing inhibitory transmission in the CeA, but possibly through different pharmacodynamic mechanisms.

One of the most important findings of this study was the differential effects of gabapentin on CeA neuronal activity in ethanol-dependent versus ethanol-naive rats. Brain slices prepared from rats continuously exposed to ethanol vapor for 2-4 weeks (ethanol-dependent rats) before electrophysiological recording exhibited an increase in baseline GABA transmission compared with nondependent rats, a trend that was further amplified via acute application of ethanol [Roberto et al. (2008), their Fig. 3B,C (http://www. jneurosci.org/cgi/content/full/28/22/ 5762/F3)]. After superfusion with gabapentin, brain slices from ethanol-dependent rats exhibited a decrease in GABA transmission [Roberto et al. (2008), their Fig. 3 (http://www.jneurosci.org/cgi/ content/full/28/22/5762/F3)], contrasting directly with the gabapentin associated increase in GABA transmission in nondependent rats. This result suggests a markedly different effect of gabapentin depending on previous ethanol exposure. Importantly, this differential reactivity to gabapentin was associated with a complementary insensitivity of GABA transmission in ethanol-dependent rats with respect to the effects of a $\mathrm{GABA}_{B}$ agonist or antagonist compared with nondependent rats [Roberto et al. (2008), their Fig. 4 (http://www.jneurosci.org/cgi/content/ full/28/22/5762/F4)]. Together, these results indicate that there are regulatory changes in the expression of $\mathrm{GABA}_{\mathrm{B}}$ receptors and/or changes in receptor sensitivity in ethanol-dependent rats.

Behaviorally, Roberto et al. (2008) clearly demonstrated the usefulness of gabapentin in the treatment of acute ethanol withdrawal using animal models of ethanol consumption and anxiety. Rats continuously exposed to ethanol (as vapor or as a liquid diet) predictably increased ethanol self-administration during acute withdrawal compared with nondependent rats [Roberto et al. (2008), their Fig. 5A (http://www.jneurosci.org/ cgi/content/full/28/22/5762/F5)]. Significantly, this effect was normalized through application of gabapentin systemically or directly into the CeA. In addition, systemic gabapentin dose-dependently blocked the anxiogenic-like effect elicited by ethanol withdrawal [Roberto et al. (2008), their Fig. 6 (http://www.jneurosci. org/cgi/content/full/28/22/5762/F6)]. The authors suggest that gabapentin relieves anxiety and consequently normal-

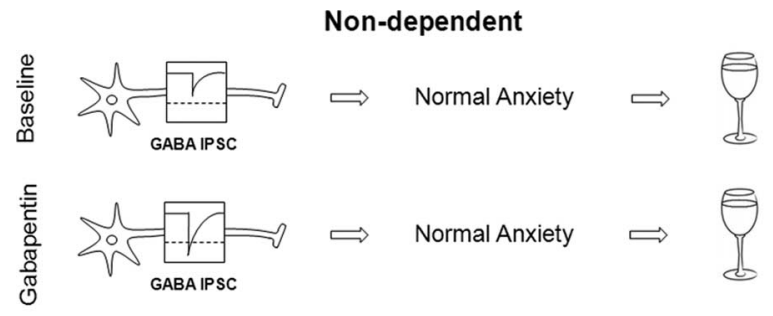

Ethanol-dependent

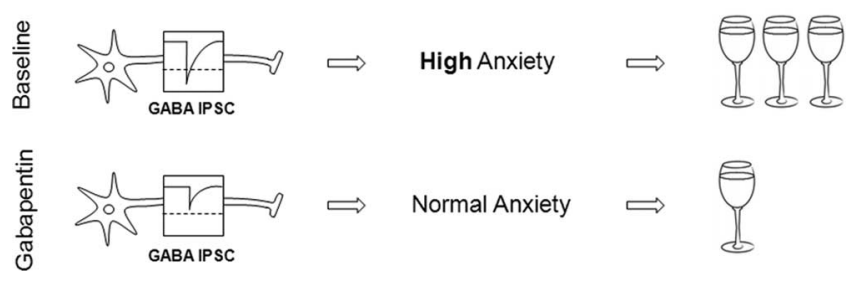

Figure 1. Rats having no previous experience with alcohol (nondependent) show "normal" levels of GABAergic transmission, as measured by the amplitude of IPSCs in the central amygdala. This is reflected in normal levels of anxiety and moderate ethanol drinking. In nondependent rats, gabapentin increases GABA transmission (increased IPSC amplitude), yet has no significant effect on behavior. In contrast, rats continuously exposed to ethanol (ethanol-dependent) have increased baseline levels of GABA transmission, display higher levels of anxiety, and consume more ethanol during acute withdrawal. Significantly, gabapentin in ethanol-dependent rats normalizes GABA transmission (i.e., reduces IPSCs to levels similar to nondependent rats at baseline), relieves the highly anxious state, and reduces ethanol consumption during withdrawal.

izes ethanol intake through its actions on GABA transmission in the CeA (Fig. 1).

Overall, this study provides strong evidence of relevant cellular and behavioral effects of gabapentin in ethanoldependent rats, yet there exist some interesting aspects that warrant further research. First, the link between GABA transmission in the CeA and anxiety in this study is quite puzzling. For example, in ethanol-dependent rats, acute ethanol increased GABA transmission, whereas gabapentin decreased GABA transmission, yet behaviorally, the two drugs displayed almost identical effects on anxiety (i.e., an anxiety-relieving effect). A similar dichotomy exists in nondependent rats: both gabapentin and ethanol increased GABA release to a similar extent, yet ethanol reduced anxiety after acute administration in naive rats (Da Silva et al., 2005), whereas acute gabapentin has no discernable effect on anxiety. If anxiety is mediated through GABA transmission in the $\mathrm{CeA}$, we would have expected gabapentin and ethanol to have similar effects on this behavior. Discrepant cellular and behavioral results may be attributable to differences in the selectivity/affinity that different treatments may have on subpopulations of GABA neurons (as discussed by the authors), but may also highlight a limitation in linking specific cellular and behavioral effects. It should also be noted here that whereas the effects of gabapentin on GABAergic transmission were studied in rats chronically ex- posed to ethanol, the effects on anxietyrelated behavior were evaluated in naive rats injected with a single high dose of ethanol. Withdrawal symptoms observed in dependent rats and in rats acutely injected with ethanol may differ markedly in terms of cellular mechanisms.

The second point for consideration here is that relapse to ethanol drinking most frequently occurs weeks to months after the cessation of drinking (protracted abstinence), rather than during the first days of abstinence (acute withdrawal). In this study, the cellular and behavioral effects of gabapentin were tested during acute ethanol withdrawal. In this period, gabapentin may have clear advantages relative to benzodiazepines considering that gabapentin is safer in terms of drug-drug interactions and hepatotoxicity, and does not exhibit addictive properties. However, the development of medications targeting protracted abstinence is a more challenging issue in drug addiction research. Hence, it would be of particular interest to investigate the cellular and pharmacological properties of gabapentin on GABA transmission and behavior [as demonstrated by Roberto et al. (2008)] at different time points after ethanol withdrawal.

Third, this research clearly highlights gabapentin as a promising drug for future treatment of ethanol dependence, but also provides valuable clues toward a more comprehensive understanding of the cellular basis of ethanol dependence. Like 
gabapentin, CRF (Heilig and Koob, 2007) or NPY (Pandey et al., 2008) systems have been implicated in anxiety-like behavior and ethanol intake in rodents. However, it is not clear whether drugs targeting these systems work on the same cellular mechanisms (GABA transmission in the CeA) or whether these drugs work on separate mechanisms but produce the same effect behaviorally. If these drugs share similar properties of altering GABA transmission in the CeA, this strengthens the hypothesis that this pathway is integral to alcohol dependence. Alternatively, if they work through separate cellular mechanisms, this may provide direction for the development of different pharmacological approaches to address alcohol dependence.

In conclusion, gabapentin "normalizes" GABAergic transmission in the CeA and reduces anxiety-like behavior and ethanol intake in rodent models of ethanol dependence. However, three major questions remain to be answered: (1) are the behavioral effects of gabapentin a direct consequence of its action on the GABAergic neurotransmission in the CeA? (2) would gabapentin also be effective in reducing ethanol drinking and anxiety during protracted withdrawal? and (3) do gabapentin and other drugs effective in reducing behavioral aspects of ethanol dependence share similar cellular properties? Further investigation of these points may contribute to our understanding of the molecular mechanisms underlying ethanol dependence and constitute an important step toward the development of medications more specific and efficacious and with less adverse effects for the treatment of alcoholism.

\section{References}

Da Silva GE, Vendruscolo LF, Takahashi RN (2005) Effects of ethanol on locomotor and anxiety-like behaviors and the acquisition of ethanol intake in Lewis and spontaneously hypertensive rats. Life Sci 77:693-706.

Furieri FA, Nakamura-Palacios EM (2007) Gabapentin reduces alcohol consumption and craving: a randomized, double-blind, placebocontrolled trial. J Clin Psychiatry 68:1691-1700.

Heilig M, Koob GF (2007) A key role for corticotropin-releasing factor in alcohol dependence. Trends Neurosci 30:399-406.

Koob GF (2003) Alcoholism: allostasis and beyond. Alcohol Clin Exp Res 27:232-243.

Pandey SC, Ugale R, Zhang H, Tang L, Prakash A (2008) Brain chromatin remodeling: a novel mechanism of alcoholism. J Neurosci 28: 3729-3737.

Roberto M, Madamba SG, Moore SD, Tallent MK, Siggins GR (2003) Ethanol increases GABAergic transmission at both pre- and postsynaptic sites in rat central amygdala neurons. Proc Natl Acad Sci USA 100: 2053-2058.

Roberto M, Gilpin NW, O'Dell LE, Cruz MT, Morse AC, Siggins GR, Koob GF (2008) Cellular and behavioral interactions of gabapentin with alcohol dependence. J Neurosci 28: 5762-5771. 